\title{
One-way Rayleigh-Wood anomalies and tunable narrowband transmission in photonic crystal gratings with broken structural symmetry
}

\author{
Andriy E. Serebryannikov ${ }^{1, *}$ and Ekmel Ozbay ${ }^{2}$ \\ ${ }^{1}$ Technische Universität Hamburg-Harburg, E-3, D-21071 Hamburg, Germany \\ ${ }^{2}$ Nanotechnology Research Center, Department of Physics, Department of Electrical Engineering, Bilkent University, 61800 Ankara, Turkey
}

(Received 28 February 2013; published 3 May 2013)

\begin{abstract}
In photonic crystal (PC) gratings whose structural symmetry is broken owing to one-side corrugations, the Rayleigh-Wood anomalies can appear in a one-way manner. In the combination with the vanishing coupling to the lowest Floquet-Bloch mode at the upper band edge, these anomalies lead to the appearance of the defect-mode-like, i.e., very narrow one-way transmission, peak, which cannot appear in the corresponding slab of PC without corrugations. Such a sole peak can be transformed into a one-way transmission band with a desired width by varying angle of incidence. The nonoverlapping and thus switchable one-way (higher-order) and two-way (zero-order) transmission bands can be obtained in both the frequency and incidence-angle domains. Adjustment of PC lattice and corrugation parameters allows one to obtain two subsequent one-way bands, every being connected with a certain higher diffraction order. Conditions required for the appearance of a narrow one-way transmission band in the vicinity of a Rayleigh-Wood anomaly are well consistent with typical dispersion features of a very large class of PCs and do not need unusual performances.
\end{abstract}

DOI: 10.1103/PhysRevA.87.053804

PACS number(s): 42.25.Fx, 42.25.Bs, 41.20.Jb, 42.70.Qs

Asymmetric transmission is a rather general phenomenon that occurs in the finite-thickness gratings with the broken spatial inversion symmetry. The interest to it has been stimulated by the attempts to find alternatives to the use of anisotropic and nonlinear materials for obtaining diodelike operation regimes. Recently, the diffraction relevant mechanism [1-3] and the polarization conversion relevant mechanism $[4,5]$ of asymmetric transmission have been studied in this concern. Nonsymmetric photonic crystal (PC) gratings [3,6] and stacked hole arrays [7] made of linear isotropic materials look promising from the wideband asymmetric transmission perspective.

Diffraction anomalies arising at a Rayleigh wavelength due to the emergence of a new outgoing diffraction order from evanescense at grazing angle that are known as Rayleigh-Wood anomalies belong to the basic features of the gratings of different types; e.g., see [8-11]. Such anomalies have first been observed by Wood [12] and then theoretically described by Rayleigh [13]. In a wide sense, Rayleigh-Wood anomalies are understood as an abrupt change of efficiency of the corresponding diffraction order. In the gratings based on pieces and slabs of periodic media like PCs, the anomalies are expected to appear in an unusual way as compared to the conventional dielectric gratings, at least because a band gap can appear in the former case. The principal possibility of one-way transmission in the vicinity of a Rayleigh-Wood anomaly has been shown in [3].

In this paper, we study asymmetric transmission in the vicinity of the first-order and second-order Rayleigh-Wood anomalies in PC gratings with one-side corrugations that are based on two-dimensional square-lattice PCs comprising circular dielectric rods. The emphasis will be put on the conditions of existence, scenarios of appearance, potential of tunability, and operation regimes associated with the filtering and switching applications. It will be shown that the

*serebryannikov@tu-harburg.de defect-mode-like narrow transmission peaks are one way due to the common effect of a Rayleigh-Wood anomaly and specific dispersion features of the two lowest Floquet-Bloch (FB) modes of the PC. In fact, the latter enables one-way appearance of the former. The width of the relevant passband can be increased by a proper variation in frequency and angle of incidence, $\theta$. Consideration is restricted to the $s$-polarized electromagnetic waves, for which the electric-field vector is parallel to the rods having diameter $d$ and permittivity $\varepsilon_{r}$. The interfaces are assumed to be along the $\Gamma-X$ direction.

According to the general theory of asymmetric transmission in PC gratings [3], equifrequency dispersion contours (EFCs) must be located around $X$ or $M$ point, or around $\Gamma$ point while being narrower than in air, in order to obtain such a wideband transmission. Similar regimes are also possible in PCs with a nonsquare lattice. For the lowest FB mode, EFCs are typically located around $M$ point at the band edge. In terms of the band diagram, this means that the frequency corresponding to $X$ point, $f=f_{1}$, is smaller than that corresponding to $M$ point, $f=f_{2}$; see Fig. 1(a). Let us assume that the band gap is located at $f_{2}<f<f_{3}$. Then, the stop band extends from $f=f_{1}$ to $f=f_{3}$ at normal incidence, $\theta=0$, but transmission can be nonvanishing at $f_{1}<f<f_{2}$ for a certain range of $|\theta|>0$.

Adjusting the PC lattice and one-side corrugation parameters, one can obtain the situation when the frequency corresponding to the -1 st-order Rayleigh wavelength, $\tilde{f}_{-1}^{R}$, satisfies the condition $f_{1}<\tilde{f}_{-1}^{R}<f_{2}$ for the period of the corrugated interface, $L>a$ ( $a$ is lattice constant), while $\tilde{f}_{-1}^{R}>f_{3}$ for the period of the noncorrugated interface, $L=a$. As a result, a Rayleigh-Wood anomaly may appear at $f=\tilde{f}_{-1}^{R}$ as a one-way transmission effect. This situation is illustrated in Fig. 1(b). Furthermore, the one-way transmission band arising in the vicinity of the anomaly may be very narrow, at least if $\tilde{f}_{-1}^{R}$ is just slightly smaller than $f_{2}$ at given $\theta$.

Figure 1(c) presents a typical example of one-way coupling at $f_{1}<f \leqslant f_{2}$ for the grating period $L=2 a$, i.e., as that in 


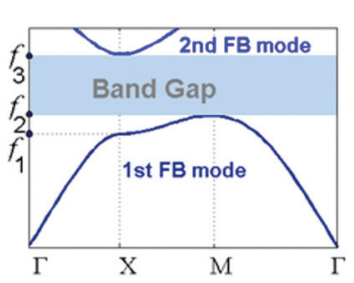

(a)

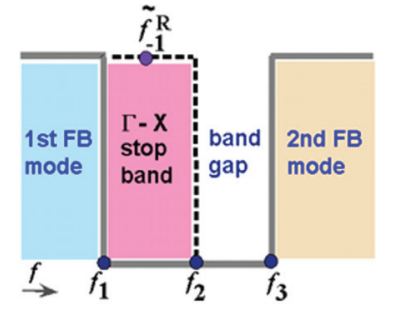

(b)

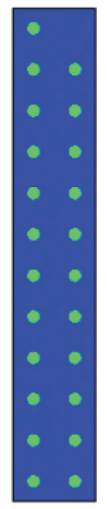

(a)

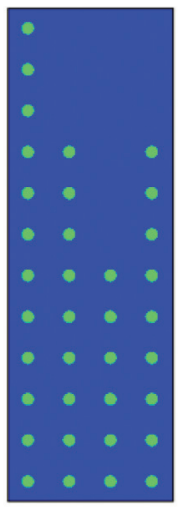

(b)

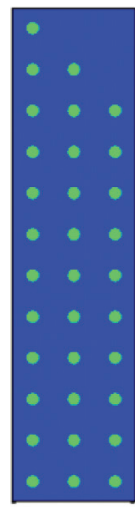

(c)

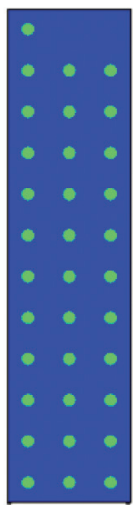

(d)

FIG. 2. (Color online) Geometry of the studied PC gratings within a grating period; the gratings shown in plots (a), (b), (c), and (d) are denoted by A, B, C, and D, respectively.

increased. In fact, this is a signature of the possible appearance of narrowband one-way transmission in the vicinity of a Rayleigh-Wood anomaly. Indeed, this means that the upper boundary of the frequency range, within which one-way transmission can appear at given $\theta, \hat{f}_{2}$, satisfies $f_{1}<\hat{f}_{2}<f_{2}$. In turn, parameters can be adjusted so that $\hat{f}_{2}=\tilde{f}_{-1}^{R}+\Delta f$, $\Delta f / \tilde{f}_{-1}^{R} \ll 1$. To compare, in Fig. 1(c), the construction line for $k L=4.78$ and $m=-1$ just touches the EFC in $\mathrm{PC}$ and EFC in air. Hence the Rayleigh-Wood anomaly is one way but singular, or pointlike, i.e., it appears only at $\phi_{-1}=-90^{\circ}$ that corresponds here to $\theta=18.3^{\circ}$. At smaller $k L$, e.g., at $k L=4.35$, there is a finite range of $\theta$ variation and, hence, a finite range of $\phi_{-1}$ variation, which starts from $-90^{\circ}$, where one-way transmission with $T^{\rightarrow}=t_{-1} \neq 0$ and $T \leftarrow=0$ takes place. Here and further, $\rightarrow$ and $\leftarrow$ stand for the cases of corrugated-side and noncorrugated-side illumination, respectively.

Geometry of the PC gratings of four types that are studied in this paper is schematically shown in Fig. 2. They have been selected based on the results of extensive simulations, by taking into account the previous experience on PC gratings [3,6,15]. Breaking spatial inversion symmetry is necessary for obtaining asymmetric transmission. Here, it is realized due to one-side corrugations. A proper choice of $L / a$ allows one to obtain the contribution of the desired higher order(s) to the one-way transmission at $f_{1}<f<\hat{f}_{2}$. The larger $L / a$, the higher is the order that may be associated with one-way transmission. Choice of the corrugation depth can be important for strength of coupling at the corrugated interface. Note that the gratings of the types A, B, C, and D show either similar or the same rod location as those in $[3,6,15,16]$, but may differ from them in values of $d / a$ and $\varepsilon_{r}$.

Figure 3 presents the maps of the symmetric $\left(t_{0}=t_{0}=\right.$ $\left.t_{0}^{\leftarrow}\right)$ and asymmetric $\left(t_{-1}\right)$ transmittances for the PC grating of type A. They illustrate the appearance and evolution of a oneway transmission band, while $t_{-1} \neq 0, t_{-1}^{\leftarrow}=0$, and $t_{0}=0$. Its lower boundary is connected with the first-order RayleighWood anomaly, whereas the upper boundary does with the peculiarities of the FB mode dispersion. The latter corresponds to $f=\hat{f}_{2}$ being dependent on $\theta$. Since these two boundaries have different slopes, the transmission band can be tuned by 

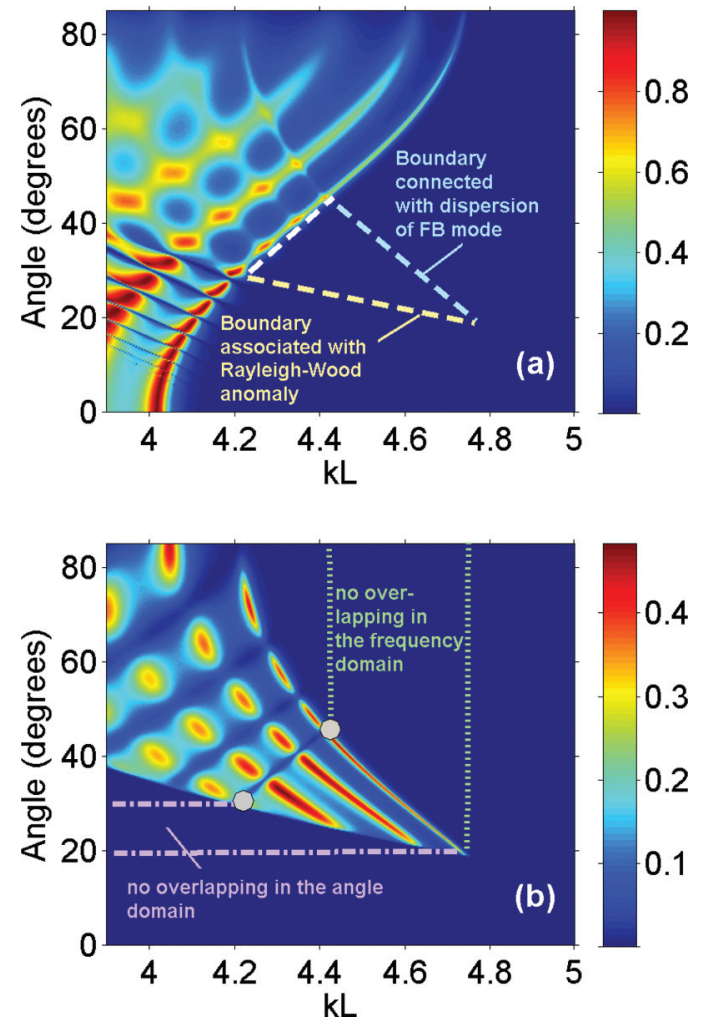

FIG. 3. (Color online) Transmittance in $(k L, \theta)$ plane for PC grating of type A at $d / a=0.4, \varepsilon_{r}=5.8, N=12$, and $L=2 a$ : (a) $t_{0}=t_{0}=t_{0}^{\leftarrow}$ and (b) $t_{-1}^{\rightarrow}$; dashed lines (a)—approximate boundary of the region of one-way transmission with $T^{\rightarrow}=t_{-1}$ and $T^{\leftarrow}=0$; dotted and dash-dotted lines (b) - boundaries of the $k L$ and $\theta$ ranges where the bands with $T^{\rightarrow}=t_{0}$ and $T^{\rightarrow}=t_{-1}$ do not overlap; circles (b) - switching between the bands with $T \rightarrow=t_{0}$ and $T^{\rightarrow}=\overrightarrow{t_{-1}}$.

means of variations in $\theta$ and/or $k L$. Furthermore, a very narrow peak of $t_{-1}$ appears near the crossing point of these boundaries, which exactly corresponds to the touching point at $k L=4.78$ in Fig. 1(c).

The boundary of the region of $t_{0} \neq 0$ in Fig. 3(a) is determined by the dispersion features presented in Fig. 1. In turn, the boundary of the region of $t_{-1}^{\leftarrow} \neq 0$ (not shown) is the same as that of $t_{0}=t_{0}^{\leftarrow} \neq 0$, because the order $m=-1$ contributes to $T \leftarrow$ due to diffractions at the exit interface $[3,16]$. One can see that the width of the one-way band with $T^{\rightarrow}=t_{-1} \neq 0$ and $T^{\leftarrow}=t_{-1}^{\leftarrow}=t_{0}=0$ can be controlled in the $f$ domain by variations in $\theta$ for a chosen value of $L$. In turn, choosing a proper value of $k L$, one can obtain a desired bandwidth in the $\theta$ domain. For a large part of the one-way transmission region in Fig. 3, the subregions in which $T^{\rightarrow}=t_{0}$ and $T \rightarrow=t_{-1}$ can be nonoverlapping, that is suitable for obtaining of switching regimes. The contrast, $C=T^{\rightarrow} / T^{\leftarrow}$, is larger than $40 \mathrm{~dB}$ over the entire region of one-way transmission in Fig. 3. Furthermore, at $k L>4.5, C$ reaches $100 \mathrm{~dB}$ in some subregions.

It is noteworthy that the mountains of $t_{-1}$ in Fig. 3(b) show negative slope, i.e., Fabry-Pérot-type behavior within the one-way transmission band occurs here similarly to the two-way transmission in the case of negative refraction in
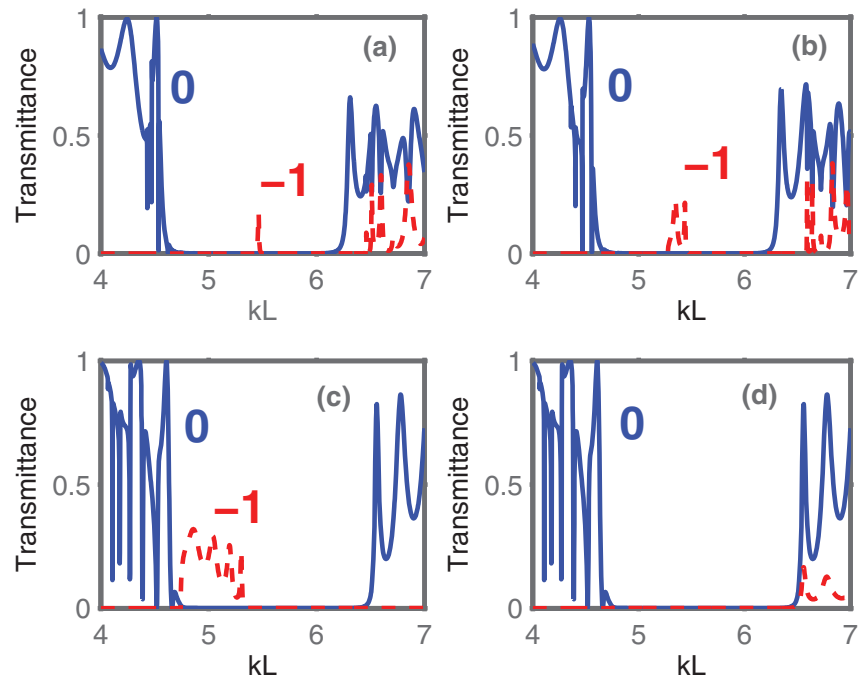

FIG. 4. (Color online) Transmittance vs $k L$ for PC grating of type A at $d / a=0.31, \varepsilon_{r}=5.8, N=12$, and $L=2 a ; \theta=9^{\circ}$ (a), $11^{\circ}$ (b), and $19^{\circ}$ (c),(d); corrugated-side (a)-(c) and noncorrugated-side (d) illumination; solid line $-m=0$; dashed line $-m=-1$.

a noncorrugated slab of PC [17]. However, in our case, it is connected with umklapp scattering rather than with the left handedness, or with taking interfaces along the $\Gamma-M$ direction for a proper EFC shape at the right handedness, as in [14]. For the values of $k L$ and $\theta$, at which $t_{0} \neq 0$ and $t_{-1} \neq 0$ simultaneously, redistribution of the transmitted energy between $t_{0}$ and $t_{-1}^{\rightarrow}$ leads to the Fabry-Pérot type nodes instead of the Fabry-Pérot mountains; compare Figs. 3(a) and 3 (b). Besides, diffraction anomalies of $t_{0}$ (they belong to the two-way anomalies) should be noticed in Fig. 3(a) at small values of $k L$ and $\theta$ that correspond to $t_{-1}=0$ in Fig. 3(b). They appear when the EFCs are already located around $M$ point, but the order $m=-1$ is not coupled yet. Their study is beyond the scope of this paper.

Figure 4 illustrates the appearance of a very narrow one-way passband associated with the Rayleigh-Wood anomaly and its evolution at varying $\theta$. Here, a smaller value of $d / a$ has been used than in Figs. 1 and 3, in order to shift the anomaly relevant, sharp one-way transmission peak towards the middle of the stop band, arising for the corresponding noncorrugated PC and for the same PC grating but at the noncorrugatedside illumination. In Fig. 4(a), it is located near $k L=5.46$ at $\theta=9^{\circ}$, where $\phi_{-1} \approx-84^{\circ}$. Here, $t_{0}=0, T^{\leftarrow}=0$, and $T^{\rightarrow}=$ $t_{-1}$. Increase of $\theta$ results in the widening of this passband and its shift towards smaller $k L$, in line with the features observed in Fig. 3. In Fig. 4(b), the bandwidth reaches 3.2\%, while $\phi_{-1}$ at the one-way band edges is varied from $-88^{\circ}$ at $k L=5.28$ to $-74.2^{\circ}$ at $k L=5.45$. In Fig. 4(c), switching between the ranges of $T^{\rightarrow}=t_{0}$ and $T^{\rightarrow}=t_{-1}$ is obtained near $k L=4.74$. For the comparison, $T^{\leftarrow}, t_{0}^{\leftarrow}$, and $t_{-1}^{\leftarrow}$ are shown in this case in Fig. 4(d). It is worth noting that the values of $\max T^{\rightarrow}=\max t_{-1}$ for the PC grating in Fig. 3 can be larger than for that in Fig. 4, while $T^{\leftarrow}=0$. For example, in Fig. 3, $T^{\rightarrow}=t_{-1}=0.33$ for the sharp peak arising at $k L=4.7$ in the vicinity of $\theta=20^{\circ}$. At $k L<4.5, \max T^{\rightarrow}=\max t_{-1}>0.4$ inside the one-way transmission band. The switching takes place near $\theta=30^{\circ}$, being similar to that in Fig. 4(c). 

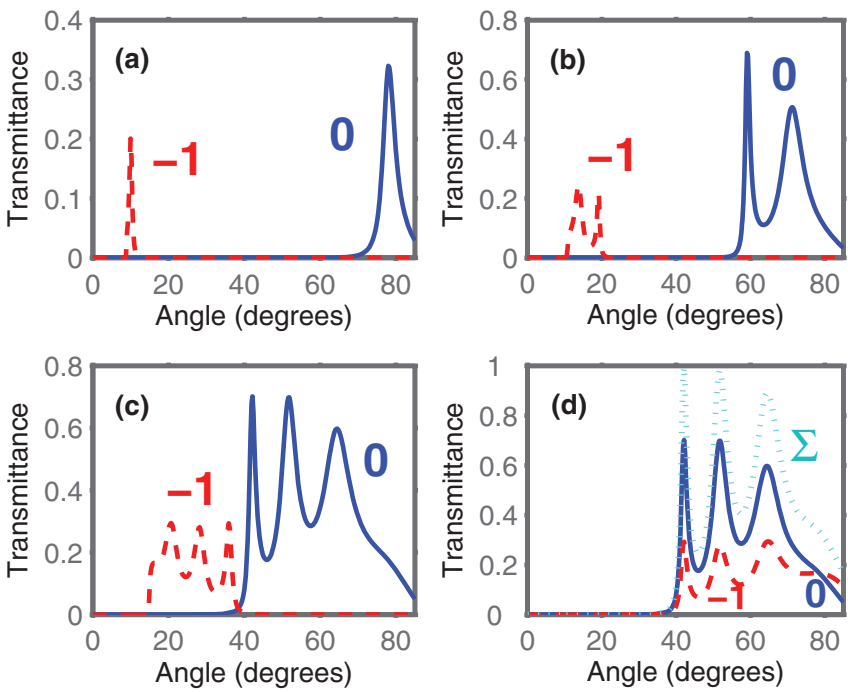

FIG. 5. (Color online) Transmittance vs $\theta$ for the same PC grating as in Fig. 4; $k L=5.45$ (a), 5.3 (b), and 5.0 (c,d); corrugated-side (a)-(c) and noncorrugated-side (d) illumination; solid line- $m=0$; dashed line $-m=-1$; (d) dotted line (denoted by $\Sigma$ )—sum over the propagating orders.

Now let us consider the one-way transmission band arising in the vicinity of the first-order anomaly in the $\theta$ domain. An example is presented in Fig. 5. The values of $k L$ are chosen so that the EFCs are located around $M$ point. In Fig. 5(a), a one-way defect-mode-like transmission peak is observed near $\theta=10^{\circ}$. This result is in agreement with the general theory of gratings, which predicts that the order $m=-1$ propagates starting from $\theta=\theta_{-1}^{R}=8.8^{\circ}$. As seen in Figs. 5(b) and 5(c), decrease of $k L$ results in widening of this transmission band. At $k L=5$ and $\theta=38.5^{\circ}$, the switching between the one-way band and the two-way band where $T \rightarrow=t_{0}=t_{0}^{\leftarrow}=t_{0}$ can be obtained. In Fig. 5(d), $T^{\leftarrow} \neq 0$ only in the $k L$ ranges where $t_{0} \neq 0$. At $\theta>38.5^{\circ}, T^{\leftarrow}>T^{\rightarrow}$ because $T^{\leftarrow}=t_{0}^{\leftarrow}+t_{-1}^{\leftarrow}$.

From the point of view of spatial filtering [18-21], the $\theta$ dependence of $T^{\rightarrow}=T^{\leftarrow}$ for the corresponding noncorrugated slab of PC (not shown) corresponds to a high-pass filter. In turn, if the PC grating is illuminated from the corrugated side, a hybrid regime can appear, in which one-way bandpass filtering at intermediate $\theta\left(T^{\rightarrow}=t_{-1}, T^{\leftarrow}=0\right)$ coexists with two-way, asymmetric transmission associated, high-pass filtering at large $\theta\left(T^{\rightarrow}=t_{0}, T^{\leftarrow}=t_{0}+t_{-1}^{\leftarrow}\right)$; see Figs. 5(c) and 5(d). Then, if the PC grating is illuminated from the corrugated and noncorrugated sides simultaneously, zero-order high-pass filtering that does not depend on the illumination side coexists with the -1 st-order relevant filtering, which is either bandpass or high pass, depending on whether the corrugated or the noncorrugated side is illuminated. Hence the nonsymmetric PC gratings operating near a Rayleigh-Wood anomaly suggest new regimes for spatial filtering.

Increase of $L / a$ allows one to obtain similar effects as those observed in Figs. 3-5 for $m=-1$, but at larger $|m|$. Figure 6 demonstrates the narrowband one-way transmission with $T^{\rightarrow}=t_{-2}$ and $T^{\leftarrow}=0$. It is seen that $\max T^{\rightarrow}$ in the one-way regime can be larger than in Figs. 3-5. For example, $\max T^{\rightarrow}=0.7$ at $k L=9.15$ in Fig. 6(b), where $\phi_{-1}=-69^{\circ}$.
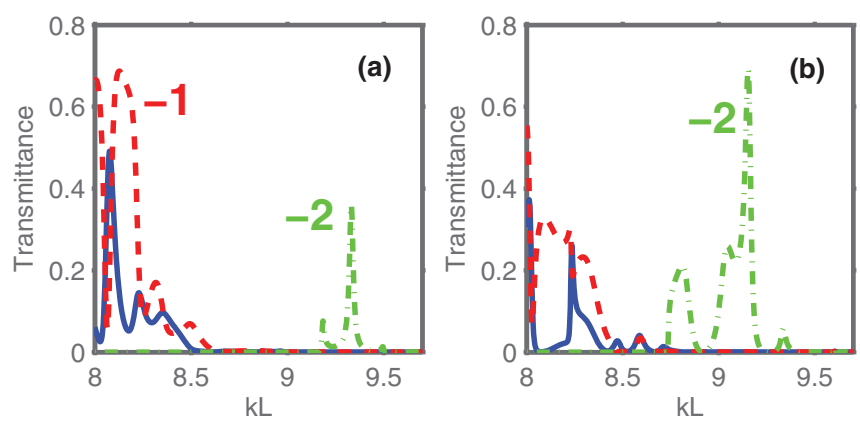

FIG. 6. (Color online) Transmittance vs $k L$ for PC grating of type B with $d / a=0.31, \varepsilon_{r}=5.8, N=12$, and $L=4 a ; \theta=21.9^{\circ}$ (a) and $26^{\circ}$ (b); corrugated-side illumination; solid line $-m=0$; dashed line $-m=-1$; dash-dotted line $-m=-2$.

Here, the order $m=-2$ becomes propagating at $k L=8.74$, being coupled to the lowest FB wave.

One can also obtain the situation when a sole diffraction order contributes to $T^{\rightarrow}$ within several subsequent ranges of $k L$ variation, which are well separated from each other by the ranges of $T^{\rightarrow}=0$. Figure 7 presents $t_{m}$ vs $k L$ in the case of such passbands with $T^{\rightarrow}=t_{0}, T^{\rightarrow}=t_{-2}$, and $T^{\rightarrow}=t_{-1}^{\rightarrow}$. Note that $T^{\leftarrow}=0$ in the second and third of them, while $T^{\rightarrow}=T^{\leftarrow}=t_{0}$ in the first one. The observed sequence differs from the conventional one, i.e., the order $m=-2$ appears at smaller $k L$ than the order $m=-1$ does. The narrow one-way transmission band in the vicinity of the Rayleigh-Wood anomaly is connected here with the order $m=-2$. Its evolution occurring with increase of $\theta$ is similar to that observed in Fig. 4 for the one-way band with $T^{\rightarrow}=t_{-1}^{\rightarrow}$ and $T^{\leftarrow}=0$. In Fig. 7, the -1 st-order relevant one-way transmission band starting in the vicinity of $k L=9.25$ is connected with the specific dispersion of the second lowest FB mode but not with the corresponding anomaly. It is noteworthy
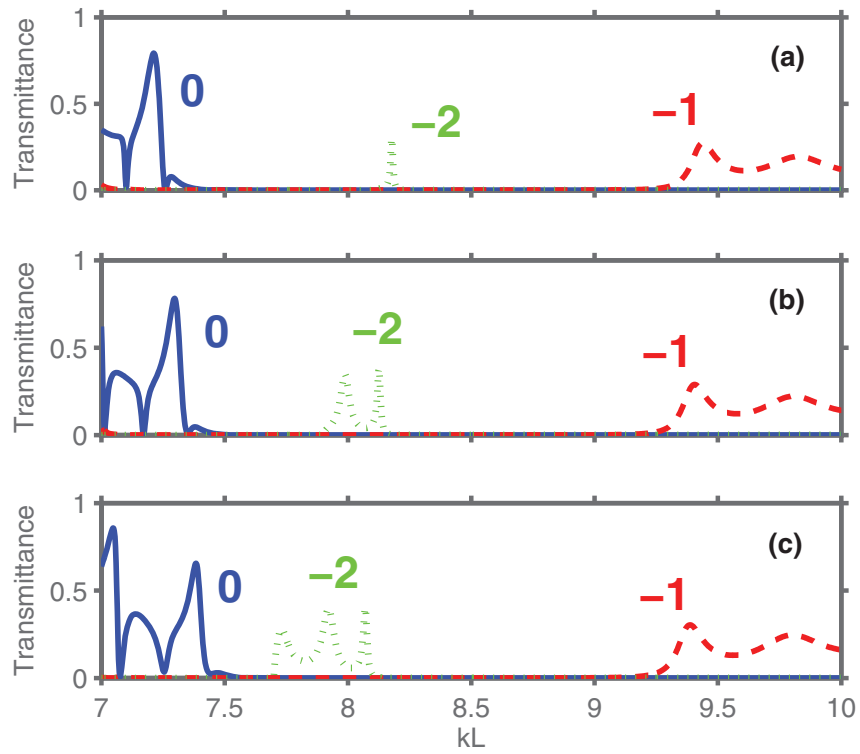

FIG. 7. (Color online) Transmittance vs $k L$ for PC grating of type C with $d / a=0.31, \varepsilon_{r}=5.8, N=12$, and $L=3 a ; \theta=33.2^{\circ}$ (a), $36.2^{\circ}(\mathrm{b})$, and $39.2^{\circ}$ (c); corrugated-side illumination; solid line$m=0$; dashed line $-m=-1$; dotted line $-m=-2$. 


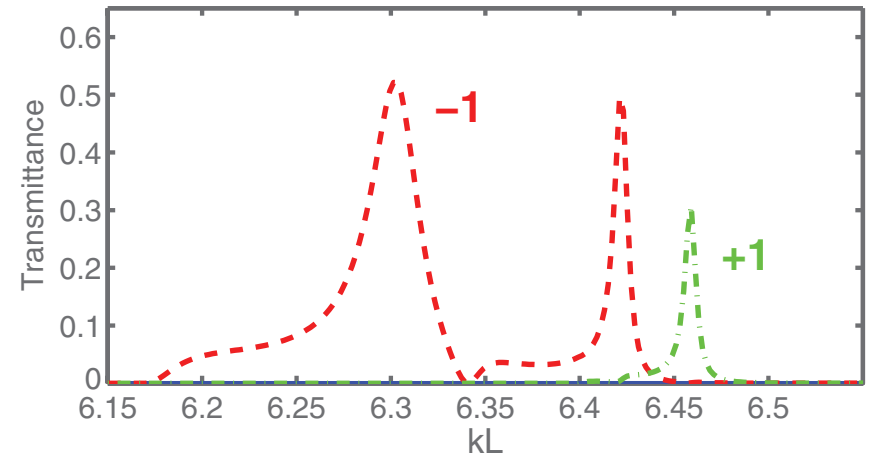

FIG. 8. (Color online) Transmittance vs $k L$ for PC grating of type D with $d / a=0.31, \varepsilon_{r}=9.61, N=12$, and $L=3 a$, at $\theta=$ $1.046^{\circ}$; corrugated-side illumination; dashed line $-m=-1$; dashdotted line $-m=+1$.

that, in contrast with the gratings of the types A, B, and D, $t_{m}$ depends here on $\operatorname{sgn} \theta$ if $|m|>0$. However, locations of the one-way bands are kept.

Finally, Fig. 8 shows the two narrow, one-way, adjacent transmission bands with $T^{\rightarrow}=t_{-1}$ and $T^{\rightarrow}=t_{+1}$ that appear at small values of $\theta>0$, while $T^{\leftarrow}=0$. One can see that even a slight deviation of $\theta$ from zero can be sufficient to avoid an overlapping of such bands, on the one hand, but is still small enough to obtain a stop band between the -1 st- and +1 st-order relevant bands, on the other hand.

To summarize, one-way transmission regimes arising in vicinity of a low-order Rayleigh-Wood anomaly due to the lowest FB wave have been studied in the structurally nonsymmetric gratings that are based on rod-type square-lattice dielectric PCs. The first and second negative order Rayleigh-Wood anomalies can manifest themselves in a one-way manner, arising at the corrugated-side illumination and vanishing at the noncorrugated-side illumination. For the latter, the existense of the band gap may lead to the situation when the same orders start contributing to the transmission at the frequencies, which are substantially higher than those corresponding to the Rayleigh cutoffs. Choosing the period of one-side corrugations, a higher-order one-way transmission band, which is connected with the Rayleigh-Wood anomaly, can be obtained at lower frequencies than a lower-order one and well separated from it. The nonoverlapping one-way bands, every one corresponding to a certain order, can occur even at weak tilting. The required conditions are very simple: the band gap must appear between the first and second lowest bands, while transmission is forbidden for the $\Gamma-X$ direction but allowed for $X-M$ and $M-\Gamma$ directions. The width of such a one-way transmission band, which may comprise several alternating Fabry-Pérot-type transmission maxima and minima, is strongly sensitive to the incidence angle variation. Possible applications include tunable one-way bandpass filters, switchers between one-way and two-way transmission bands, and spatial filters that combine one-way and two-way bands.

This work is supported by the projects DPT-HAMIT, ESF-EPIGRAT, NATO-SET-181 and by TUBITAK under Projects No. 107A004, No. 109A015, and No. 109E301. A.E.S. thanks DFG for partial support of this work under Project No. SE1409/2-2. E.O. acknowledges support from the Turkish Academy of Sciences.
[1] A. E. Serebryannikov and E. Ozbay, Opt. Express 17, 13335 (2009).

[2] M. J. Lockyear, A. P. Hibbins, K. R. White, and J. R. Sambles, Phys. Rev. E 74, 056611 (2006).

[3] A. E. Serebryannikov, Phys. Rev. B 80, 155117 (2009).

[4] C. Menzel, C. Helgert, C. Rockstuhl, E.-B. Kley, A. Tünnermann, T. Pertsch, and F. Lederer, Phys. Rev. Lett. 104, 253902 (2010).

[5] M. Mutlu, A. E. Akosman, A. E. Serebryannikov, and E. Ozbay, Phys. Rev. Lett. 108, 213905 (2012).

[6] A. E. Serebryannikov, A. O. Cakmak, and E. Ozbay, Opt. Express 20, 14980 (2012).

[7] M. Beruete, A. E. Serebryannikov, V. Torres, M. Navarro-Cia, and M. Sorolla, Appl. Phys. Lett. 99, 154101 (2011).

[8] A. Hessel and A. A. Oliner, Appl. Opt. 4, 1275 (1965).

[9] M. Sarrazin, J.-P. Vigneron, and J.-M. Vigoureux, Phys. Rev. B 67, 085415 (2003).

[10] V. Lomakin and E. Michielssen, IEEE Trans. Antennas Propag. 55, 1564 (2007).
[11] M. Beruete, M. Navarro-Cia, V. Torres, and M. Sorolla, Phys. Rev. B 84, 075140 (2011).

[12] R. W. Wood, Philos. Mag. 4, 396 (1902).

[13] Lord Rayleigh, Proc. R. Soc. Lond. A 79, 399 (1907).

[14] C. Luo, S. G. Johnson, J. D. Joannopoulos, and J. B. Pendry, Phys. Rev. B 65, 201104(R) (2002).

[15] A. O. Cakmak, E. Colak, A. E. Serebryannikov, and E. Ozbay, Opt. Express 18, 22283 (2010).

[16] A. E. Serebryannikov, K. B. Alici, T. Magath, A. O. Cakmak, and E. Ozbay, Phys. Rev. A 86, 053835 (2012).

[17] A. E. Serebryannikov, E. Ozbay, and P. V. Usik, Phys. Rev. B 82, 165131 (2010)

[18] D. Schurig and D. R. Smith, Appl. Phys. Lett. 82, 2215 (2003).

[19] A. E. Serebryannikov, A. Y. Petrov, and E. Ozbay, Appl. Phys. Lett. 94, 181101 (2009).

[20] K. Staliunas and V. J. Sanchez-Morcillo, Phys. Rev. A 79, 053807 (2009)

[21] E. Colak, A. O. Cakmak, A. E. Serebryannikov, and E. Ozbay, J. Appl. Phys. 108, 113106 (2010). 